\title{
Particulate reduction in PLD-grown crystalline films via bi-directional target irradiation
}

\author{
Jake J. Prentice ${ }^{1}$ - James A. Grant-Jacob ${ }^{1} \cdot$ Sergey V. Kurilchik ${ }^{1} \cdot$ Jacob I. Mackenzie $^{1} \cdot$ Robert W. Eason $^{1}$
}

Received: 28 November 2018 / Accepted: 28 January 2019 / Published online: 2 February 2019

(c) The Author(s) 2019

\begin{abstract}
We present a novel variation of the pulsed laser deposition (PLD) technique, aimed at reducing the number of particulates produced and consequently the linear propagation loss observed in the resulting crystal waveguides. The approach relies upon configuring the system to effectively provide bi-directional ablation, whereby the incidence angle of the fixed pulsed laser beam with respect to the target surface changes sign, depending upon the rotation angle and position of the target. Such an alternating ablation direction is intended to reduce the buildup of undesirable periodic surface structures, such as directional cones, believed to be a major source of particulates within the growing film while keeping the plume stationary with respect to the substrate. We show that targets ablated using this technique have fewer directional structures and a decreased surface roughness. Furthermore, using PLD-grown $\mathrm{Y}_{3} \mathrm{Ga}_{5} \mathrm{O}_{12}$ as the exemplar crystal film, we compare growths with uni- and bi-directional ablation and demonstrate reduction from $\sim 0.9$ to $\sim 0.23 \mathrm{~dB} / \mathrm{cm}$ in the average waveguide propagation losses via the latter.
\end{abstract}

\section{Introduction}

Pulsed laser deposition (PLD) is a quick, versatile method for depositing thin films comprising a wide selection of materials that include single elements, such as metals, or complex multi-component materials [1, 2]. In PLD, laser pulses, usually in the UV region, ablate the target material containing the atomic constituents to be deposited onto a heated substrate. The site of the laser incidence experiences rapid heating due to the absorption of the laser energy, and for pulse durations shorter than the time required for heat diffusion, the irradiated area undergoes an ablation process leading to material being ejected from the target as a plasma plume toward the substrate, which is placed in close proximity (usually some few $\mathrm{cm}$ distant).

PLD has been demonstrated as a viable technique for the growth of single-crystal planar waveguide devices fabricated from a range of materials, including doped sapphire $\left(\mathrm{Al}_{2} \mathrm{O}_{3}\right)[3,4]$, sesquioxides $[5,6]$ and in particular, garnets [7]. This can be a relatively fast deposition process, and to date, growth rates of $25-\mu \mathrm{m}$ per hour have been achieved on

Jake J. Prentice

J.J.Prentice@soton.ac.uk

1 Optoelectronics Research Centre, University of Southampton, Highfield, Southampton SO17 1BJ, UK 1-cm² samples [8]. PLD-grown Yb:YAG (yttrium aluminum garnet), as the gain medium in a laser, has recently delivered $>21 \mathrm{~W}$ output power with $70 \%$ slope efficiency [9], a new record for waveguide lasers fabricated by this approach. Furthermore, other active garnet films, including Yb:GGG (gadolinium gallium garnet) and Yb:YGG (yttrium gallium garnet) have also been used for efficient laser operation [10].

Although these results are promising, a key aspect for increasing the lasing efficiency is reducing the waveguide propagation loss in the deposited film. One potential contribution to this loss [11] is particulates that are routinely embedded into the film during the deposition process. Particulates are created, in part, by the degradation of the surface of the target material, which during ablation ejects a mixture of electrons, ions, neutrals, molecules, and clusters as well as undesirable particulates that are an inevitable consequence of the PLD process. These particulates are incorporated into the growing film and contribute to scattering loss in optical waveguides. A range of techniques have been developed to reduce, or ideally prevent the production of these particulates, [11-18], but it is generally accepted within the PLD community that particulates are an inevitable by-product of the fundamental ablation process.

In this paper, we demonstrate a new ablation technique designed to reduce the surface degradation of the target, and consequently, the density of particulates in PLDgrown films. We present a simulation of the ablation path 
of consecutive pulses on the target for our configuration, showing that this leads to an effective bi-directional ablation protocol that covers most of the target surface area. To support this hypothesis, we present the characterization of the resulting surface topology of targets subjected to uni- or bidirectional ablation, demonstrating a reduction in the amplitude of directional structure for the latter, under equivalent ablation conditions. This regime also allows in excess of 1000 laser pulses per unit area before critical target damage, which means thicker films with fewer scattering points can be grown, paving the way for an additive fabrication method for active thin-disks, which require film thicknesses of typically $\sim 100 \mu \mathrm{m}$ or more, for use in the well-established thindisk-laser architecture. Dark-field microscope images of the resulting PLD-grown crystal films have further proved that there is a major reduction in the density of scattering points in those films produced via bi-directional ablation.

We are confident that this new PLD approach represents a significant advance in the growth of high-quality crystalline layers as it leads to the inclusion of significantly fewer particulates within the film. In addition, we found that target life was prolonged before reconditioning of the surface was required. Both factors are very important if PLD is to be considered as a routine process for growth of high-quality low-loss crystalline films.

\section{Surface degradation in PLD targets}

Shown in Fig. 1 is a typical PLD set-up, which illustrates some of the basic components required. Typically, targets are fabricated from powders that are compressed and

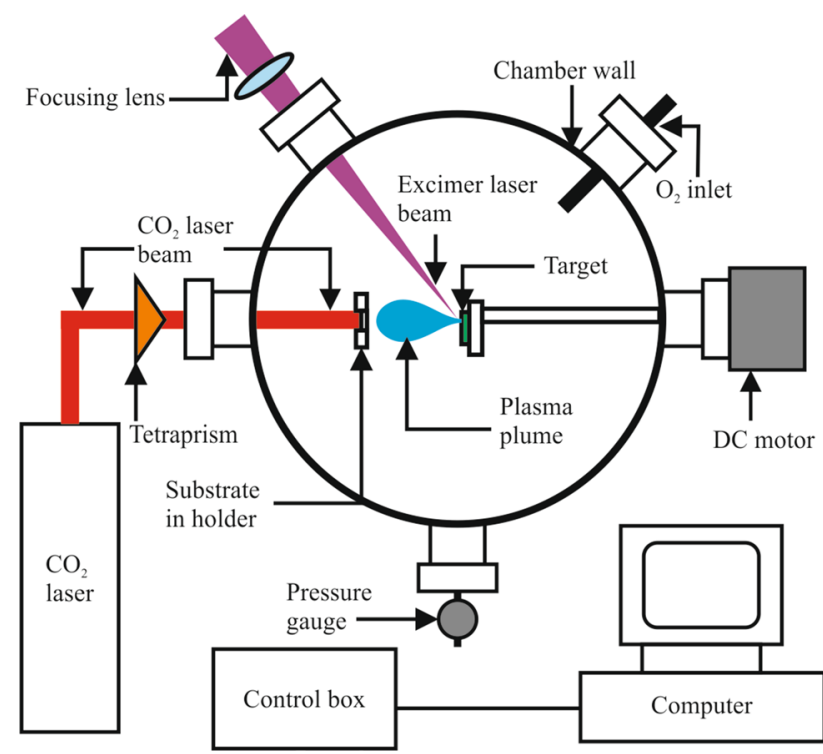

Fig. 1 Schematic of a typical single beam/single target PLD set-up sintered, leading to ceramic discs that are robust enough to be used for long periods of ablation. In general, the diameters of these discs can vary from around $25 \mathrm{~mm}$ diameter to greater than $200 \mathrm{~mm}$. They are routinely moved or rotated during exposure to the incident laser radiation to avoid overexposure to multiple pulses, which leads to severe surface degradation and the build-up of surface structures that can lead to decreasing ablation efficiency. Another major consequence of laser-induced surface structure in PLD, such as cone formation, is the production of particulates due to exfoliation from their tips. The greater the accumulated local exposure of a particular area, the more evident is the presence of these cones, which appear to lead to the transfer of $\sim 0.1 \mu \mathrm{m}$ to several- $\mu \mathrm{m}$-scale particulates from the target to the growing film. A typical example of a target surface that has been exposed to approximately 6000 pulses $/ \mathrm{cm}^{2}$ from single-direction ablation is shown in Fig. 2. This ceramic target, comprising $\mathrm{Y}_{2} \mathrm{O}_{3}$ and $\mathrm{Ga}_{2} \mathrm{O}_{3}$ powders (to be referred to as a YG target in the following), had been compressed and sintered to reach a final effective density of $>85 \%$. The rotating/translating target (as detailed in the next section) was subjected to ablation by a $\mathrm{KrF}$ excimer laser beam in a background $\mathrm{O}_{2}$ pressure of $0.02 \mathrm{mbar}$, for a period of $30 \mathrm{~min}$ at a laser repetition rate of $100 \mathrm{~Hz}$.

In Fig. 2 it is apparent that cone structures point in a preferred direction, which is toward the incident laser beam, at a $45^{\circ}$ angle of incidence with respect to the surface normal. To prevent the build-up of these undesirable structures, we have, therefore, developed and implemented an ablation configuration where the angle of incidence is periodically varied with respect to the target normal, so that every region is exposed to pulses that are incident from angles of equal and opposite sign $\left(+45^{\circ}\right.$ and $-45^{\circ}$ for example). Our aim here was to demonstrate that if there is no preferential unique angle of incidence of the laser pulse with respect to

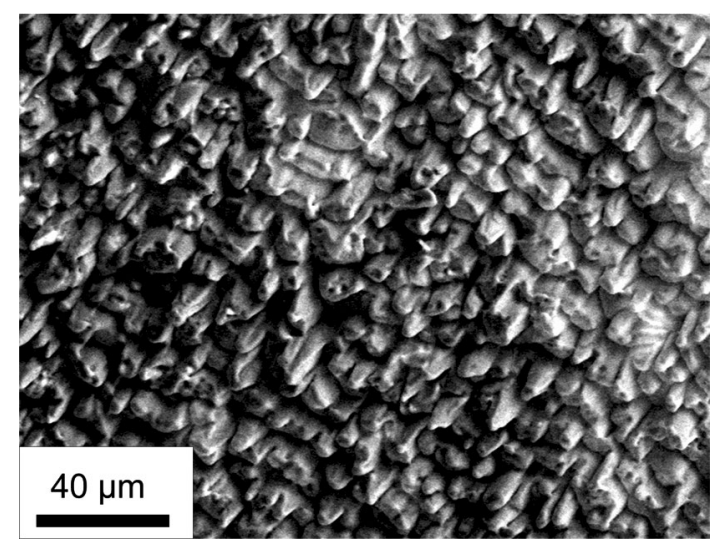

Fig. 2 SEM image of the surface of a YG ceramic target that has been exposed to approximately 6000 ablation pulses $/ \mathrm{cm}^{2}$, at a $45^{\circ}$ angle of incidence via uni-directional ablation 
the target normal, there can be no subsequent preferential build-up of cone structures pointing back to the direction of the laser pulse, as observed in Fig. 2, and hence our adoption of this novel bi-directional ablation protocol.

\section{Existing methods to reduce target surface degradation}

Figure 3 shows a simple ablation scenario where a target is rotated in front of the incident laser beam, resulting in a narrow ablation track, and hence inefficient use of the target surface. While the radial position of the incident laser pulse can routinely be adjusted during growth, and a new ablation ring exposed, this also has the consequence of spatially shifting the plume with respect to the static substrate. For many cases, this is undesirable as it can lead to varying stoichiometric transfer and reduced deposition rates.

To compensate for these problems, we have routinely implemented a technique referred to as epitrochoidal motion, whereby the target is rotated via an offset cam, and

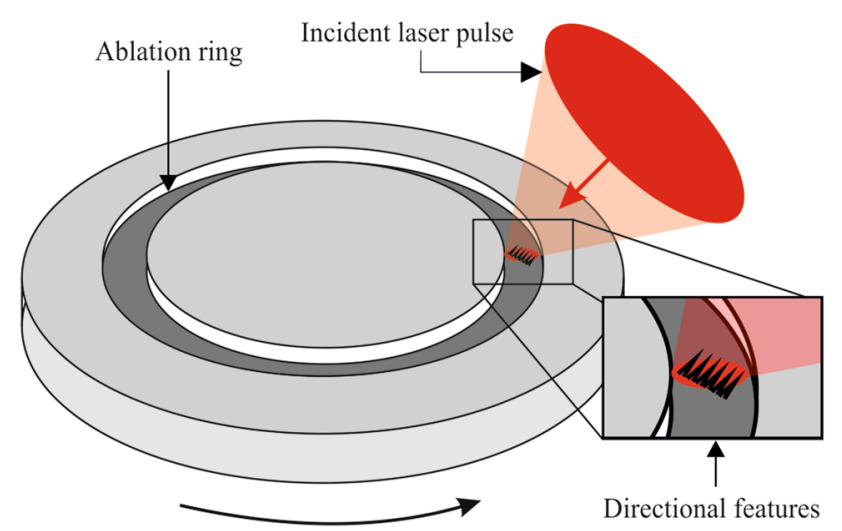

Fig. 3 Ablation track in a target under simple circular rotation geometry with the formation of cones directed radially outwards (shown in the inset) hence the area used for ablation is significantly increased beyond that for the simple geometry shown in Fig. 3. The point of incidence of the laser can be equated to a rotating spiral, as illustrated in the simulation displayed in Fig. 4, where the modelled cam and target had radii of $15 \mathrm{~mm}$ and $25 \mathrm{~mm}$, respectively, with an axial offset for the cam equal to $12.5 \mathrm{~mm}$. A photograph of the ablated target using this cam arrangement is also shown in Fig. 4.

While beneficial for making good usage of the target surface, there was no attempt here to reduce the basic problem of surface structure formation. This has been addressed by others, however, particularly in the context of large area commercial scale-up of PLD $[1,19]$.

To reduce the directionality of these cones requires symmetry-breaking, deviating away from a purely rotational motion for the target, and this can be accomplished by rotating the target periodically after raster-scanning of the laser beam across the target. Figure 5 is a schematic of the technique adopted in [19], and Fig. 5d shows a target that has been exposed to this raster scan method.

\section{Bi-directional ablation technique}

We now detail our novel technique that aims to significantly reduce or ideally remove the problem of surface feature build-up on the target, through the adoption of an alternating irradiation geometry. Our bi-directional ablation protocol is simply illustrated in Fig. 6.

For a single beam and uni-directional irradiation, conelike structures are formed as shown in Fig. 6a, as evidenced in the SEM image in Fig. 2. The mirror image would be true for an incidence angle from the opposite direction as in Fig. 6b. If irradiation is performed from both directions, either simultaneously or sequentially, as in our work reported here, shown in Fig. 6c there is no unique direction of incidence and, theoretically, there should be no possibility of direction-dependent cone formation.
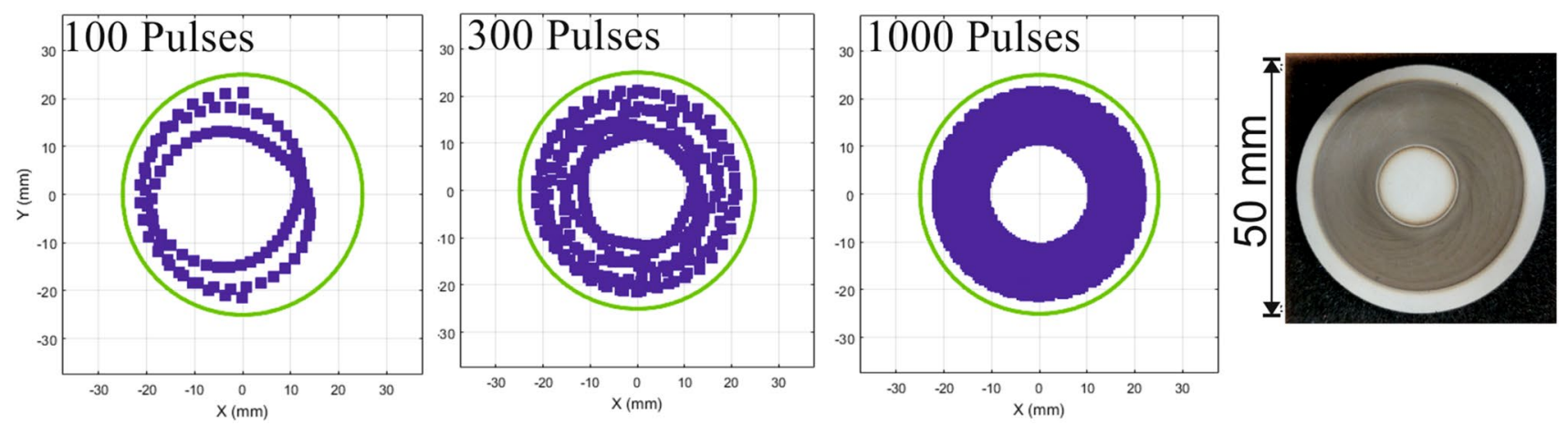

Fig. 4 Sequential progression of the ablation spot position on a 25-mm radius target undergoing epitrochoidal motion, with a cam radius and offset of $15 \mathrm{~mm}$ and $12.5 \mathrm{~mm}$, respectively. A photograph (far right) shows an ablated YG target after 36,000 shots for the same configuration 
Figure 7 shows the movement of the target with the bidirectional ablation setup; note that the target's displacement path moves the irradiated spot through the center of the target, which is key for the bi-directional ablation protocol, while ensuring the plume is stationary relative to the substrate. As mentioned previously, this is of significance for the case where high growth rates, and hence thick films, are required on relatively small substrates (in our case $1 \mathrm{~cm}^{2}$ ), as moving the plume relative to the substrate can significantly reduce the resultant film thickness, as well as introducing stoichiometric variation across the film.
A model of the ablation path on the target for the bidirectional design is presented in Fig. 8, illustrating the opposing angles of incidence on the target by the two differently colored (red and purple) squares. Ideally, there would be an equal number of shots at each point on the target from either incidence direction, $\left(+45^{\circ}\right.$ and $\left.-45^{\circ}\right)$, and this can be seen with the red squares eventually overlapping the purple ones, for the 1000 pulse case, resulting in an averaging of the ablation angle and thus reducing the build-up of directional surface stuctures.
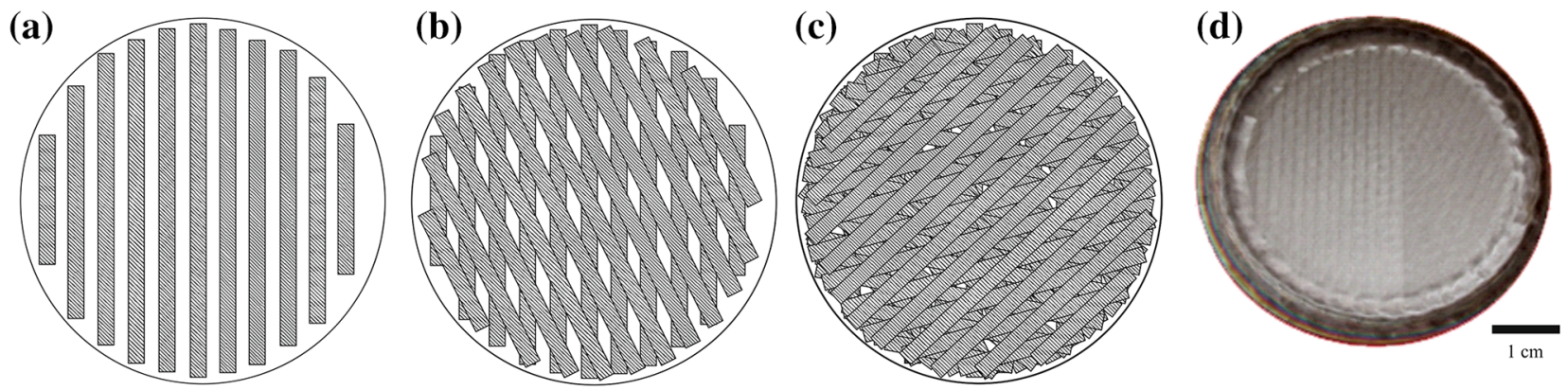

Fig. 5 Schematic showing target surface patterning for a single raster scan (a), the subsequent pattern produced after target rotation by $26.5^{\circ}(\mathbf{b})$ and after 5 such $x-y-\theta$ raster and rotation sequences $\mathbf{c}$ after

[1]. A photograph $\mathbf{d}$ of a 6-inch diameter YCBO target that has been subjected to this procedure [1] (used with permission)

(a)

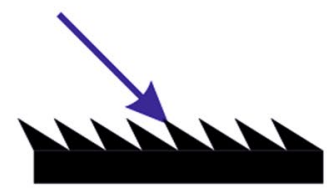

(b)

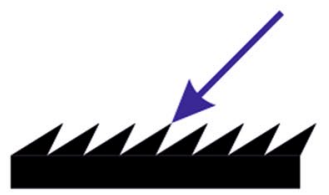

(c)

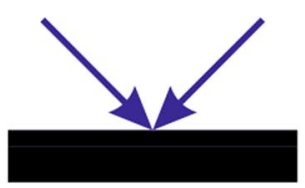

Fig. 6 a Cone production from pulses incident at one unique angle, $\mathbf{b}$ from the equal and opposite angle, and $\mathbf{c}$ the resulting ideal situation corresponding to the combination of $\mathbf{a}$ and $\mathbf{b}$ either simultaneously, or sequentially as in the case discussed in this work

(a)

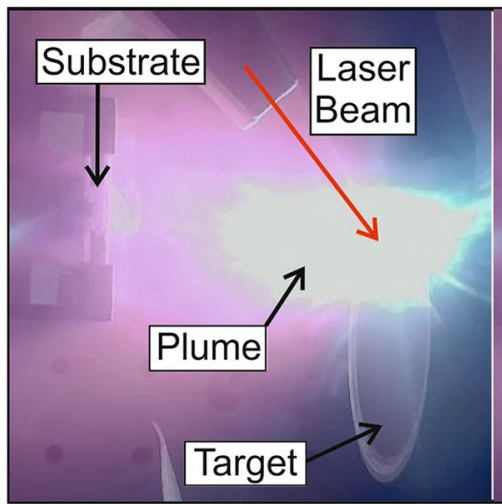

(b)

(c)

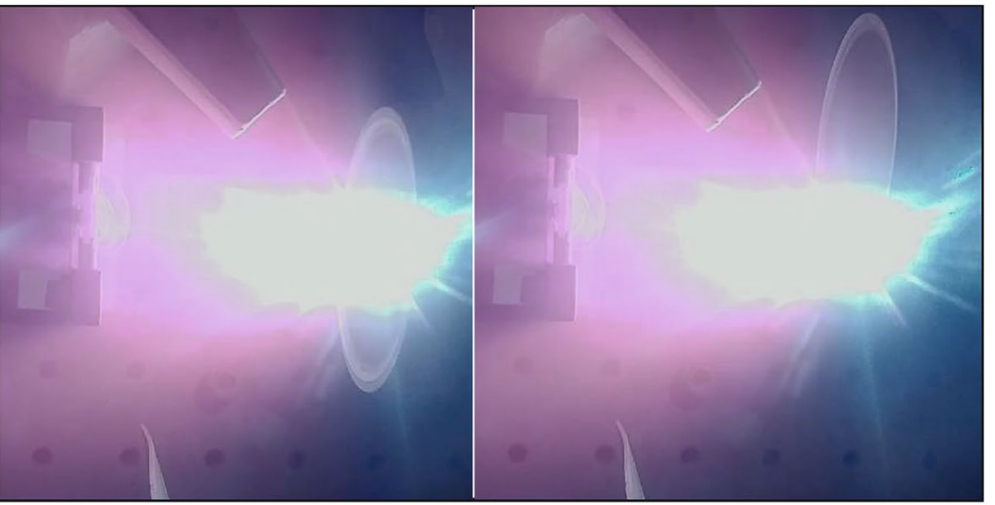

Fig. 7 Images of PLD showing the target movement using a bi-directional ablation setup. The rotating target is simultaneously translated, by almost its full diameter, from one side (a), through the centre (b) to the other side (c), while the plume remains stationary relative to the substrate 

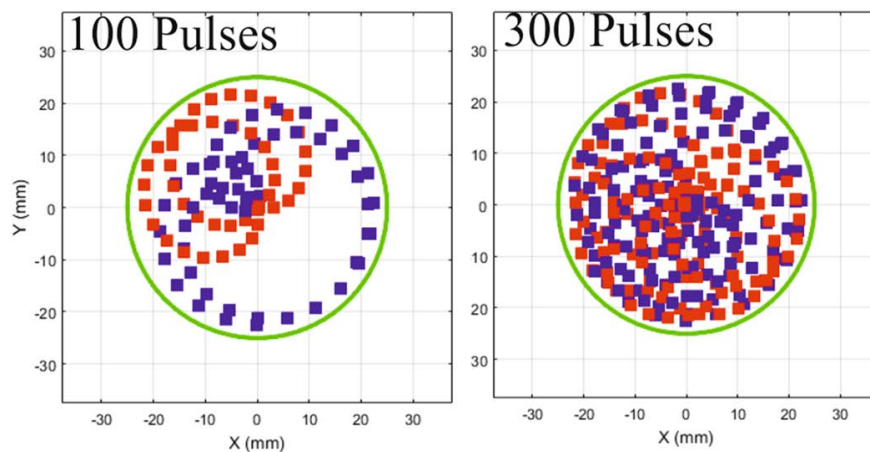

Fig. 8 Model of ablation pattern with the bi-directional ablation arrangement after 50,100, 300 and 1000 pulses. Red and purple squares indicate opposing angles of incidence for the laser. The green

\section{Results}

Figure 9 shows SEM images of an ablated YG target after $\sim 36,000$ pulses $/ \mathrm{cm}^{2}$ using the uni-directional setup on one face of the target, and the bi-directional setup on the other. With the uni-directional setup (Fig. 9a), there is a clear directionality to the surface structure and evidence of small surface particles shown in the inset, whereas bi-directional ablation (9b) while still producing some target structuring, has no obvious directionality nor evidence of surface particles.

Measurement of the target surface roughness is given in Fig. 10, which enabled comparison of the as-made unused target with regions exposed to uni- or bi-directional ablation. The peak-to-valley range shows a $\sim 50 \%$ reduction in surface roughness for bi- as compared to uni-directional ablation. Moreover, it is only a factor of 2 larger than the surface roughness present on a new and unused target. Both the reduction in directionality and scale of the surface structures is a significant improvement compared with uni-directional ablation.

Further evidence of the benefits of this new approach can be observed in dark field microscopy images, as shown in Fig. 11. These images, of two different films grown from an erbium-doped YG target, using an ablation fluence of $1.3 \mathrm{~J} /$ $\mathrm{cm}^{2}$ and $\mathrm{a} \sim 700{ }^{\circ} \mathrm{C}$ substrate temperature, in a partial $\mathrm{O}_{2}$ atmosphere at 0.02 mbar, were taken with a $100 \times$ objective. Figure 11a represents a film grown with the uni-directional ablation technique, while Fig. $11 \mathrm{~b}$ is with the new bi-directional ablation protocol.

A script was written using Python (v3.5), to determine the percentage of pixels in the dark field images that were above a defined threshold intensity (i.e. $10 \%$ of the maximum pixel intensity in the image), to determine the percentage coverage of defects in each dark field image. For the images in Fig. 11, values of $3.25 \%$ and $0.27 \%$ were determined for the uni-directional and bi-directional

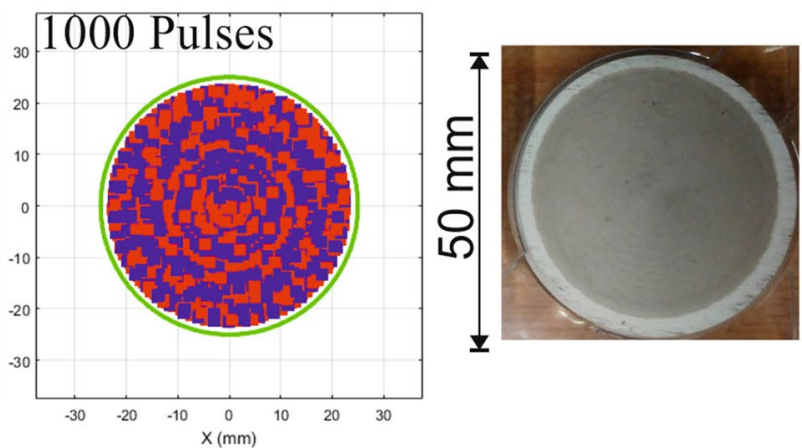

circle indicates the perimeter of the target. A photograph (far right) of a target that has undergone this procedure is also shown

(a)

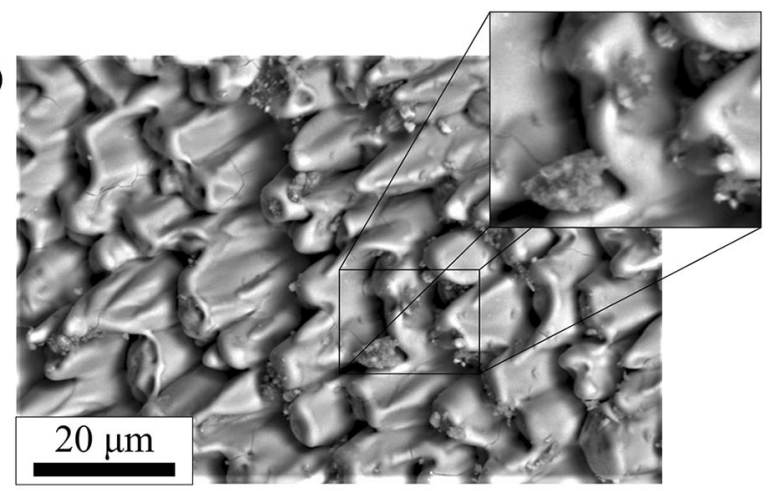

(b)

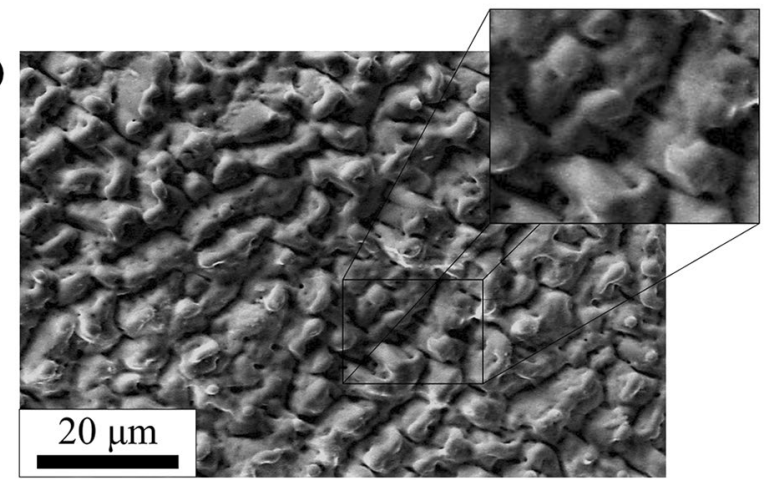

Fig. 9 SEM images of surface of an ablated YG target after irradiation with $\sim 36,000$ pulses $/ \mathrm{cm}^{2}$ using the a uni-, b bi-directional ablation protocols. The insets highlight small particles in a, which could be dislodged and deposited onto the growing film and were only observed for uni-directional ablation runs, i.e. no small particles can be found in $\mathbf{b}$

setups, respectively. Note, this measurement takes into account the quantity and size of particulates observed in a range of deposited films, for dark field images recorded at $100 \times$ magnification, as shown in a histogram for uni(Fig. 12a) and bi-directional ablation (Fig. 12b). For films grown via uni-directional ablation, the average coverage 


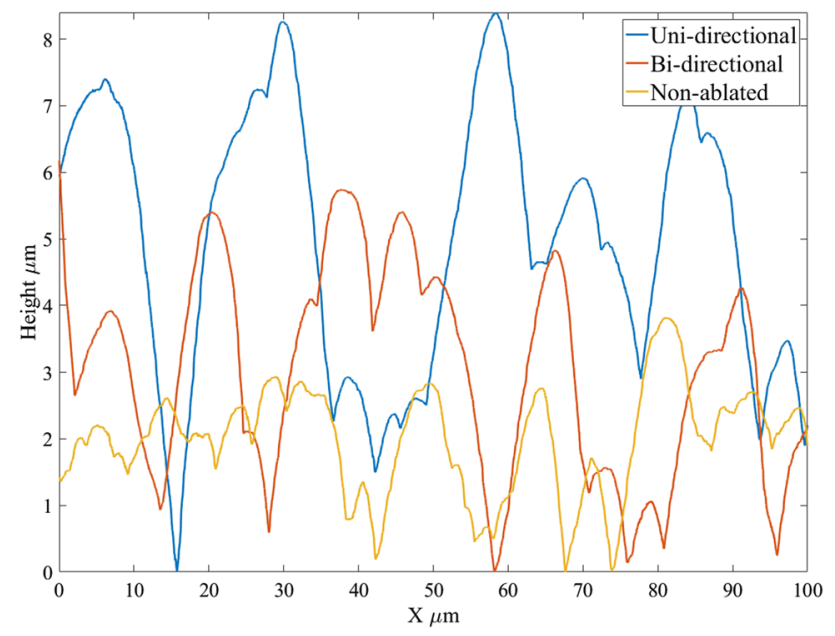

Fig. 10 Stylus profiler measurements of uni-directional ablated (blue), bi-directional ablated (orange) and non-ablated (yellow) YG targets

is $(1.0 \pm 0.22) \%$ based on 28 samples, whereas with bidirectional ablation the average coverage is $(0.17 \pm 0.03) \%$ based on 19 samples. The uncertainties in these values were calculated using the standard error. This shows an improvement by a factor of $\sim 5$ in film quality obtained utilizing this new approach. It should be noted that all growths were performed on as-manufactured or reconditioned targets that had no significant surface damage. Prior to a growth run on a new target, a pre-ablation routine is undertaken, consisting of 36,000 pulses to ensure all growths are performed with a similar target surface and any surface impurities are removed.

Furthermore, using a white light interferometer (Zescope) at 50× magnification, the surface roughness $\left(S_{\mathrm{a}}\right)$ and surface particulate density were measured. In addition, the size of the surface particulates could be quantified as shown in Fig. 13, by histograms for the number of particulates per $\mathrm{mm}^{2}$ that are higher than $10 \mathrm{~nm}, 20 \mathrm{~nm}$, and $100 \mathrm{~nm}$, respectively. Collectively, these plots demonstrate a significant reduction in surface particulates, for all sizes investigated, with the new bi-directional approach. The average as-grown film $S_{\mathrm{a}}$ values for uni- and bi-directional ablation were $(2.14 \pm 0.14) \mathrm{nm}$ and $(1.13 \pm 0.04) \mathrm{nm}$ respectively, using the standard error, showing a $50 \%$ decrease in surface roughness. These results again demonstrate the dramatic improvement in the film-surface quality obtained with the bi-directional ablation protocol.
Fig. 11 Dark field microscopy images, at $\times 100$ magnification, of films grown under the same growth conditions, via uni- (a) and bi-directional (b) ablation

Fig. 12 Histogram of the percentage of area covered by scattering points in dark field images, at $\times 100$ magnification, when grown via uni- (a) and bi-directional ablation (b) (a)

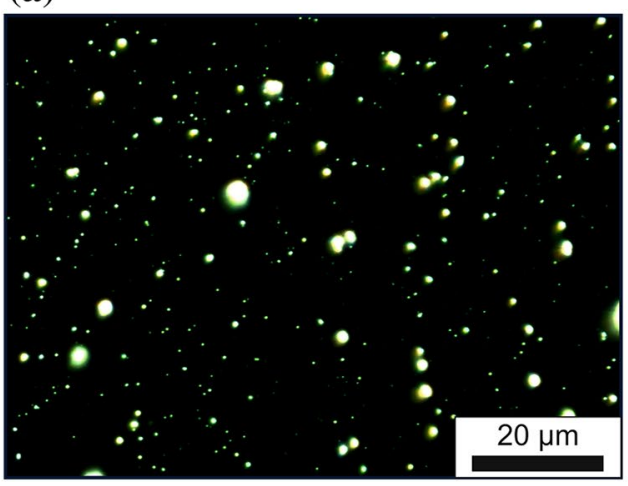

(b)

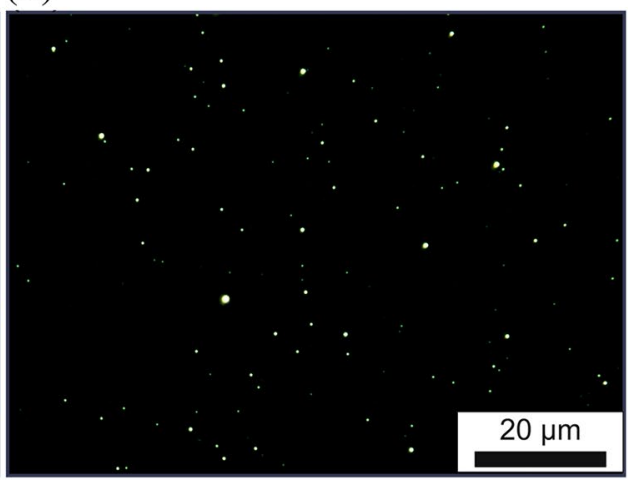

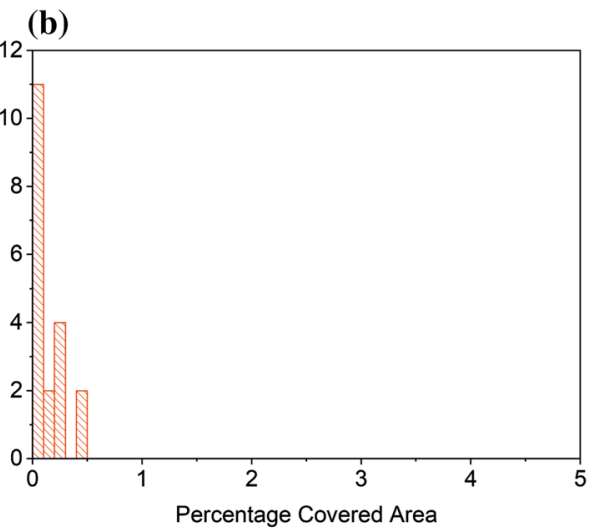


Fig. 13 Histograms of the surface particulate density (particles $/ \mathrm{mm}^{2}$ ), when counting particles with heights greater than: $10 \mathrm{~nm}$ (red diagonals), $20 \mathrm{~nm}$ (green diagonals), and $100 \mathrm{~nm}$ (purple crosses); on films grown via uni- (a) or bidirectional ablation (b) (a)

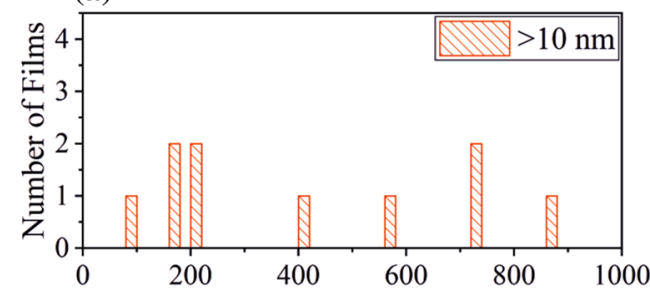

(b)
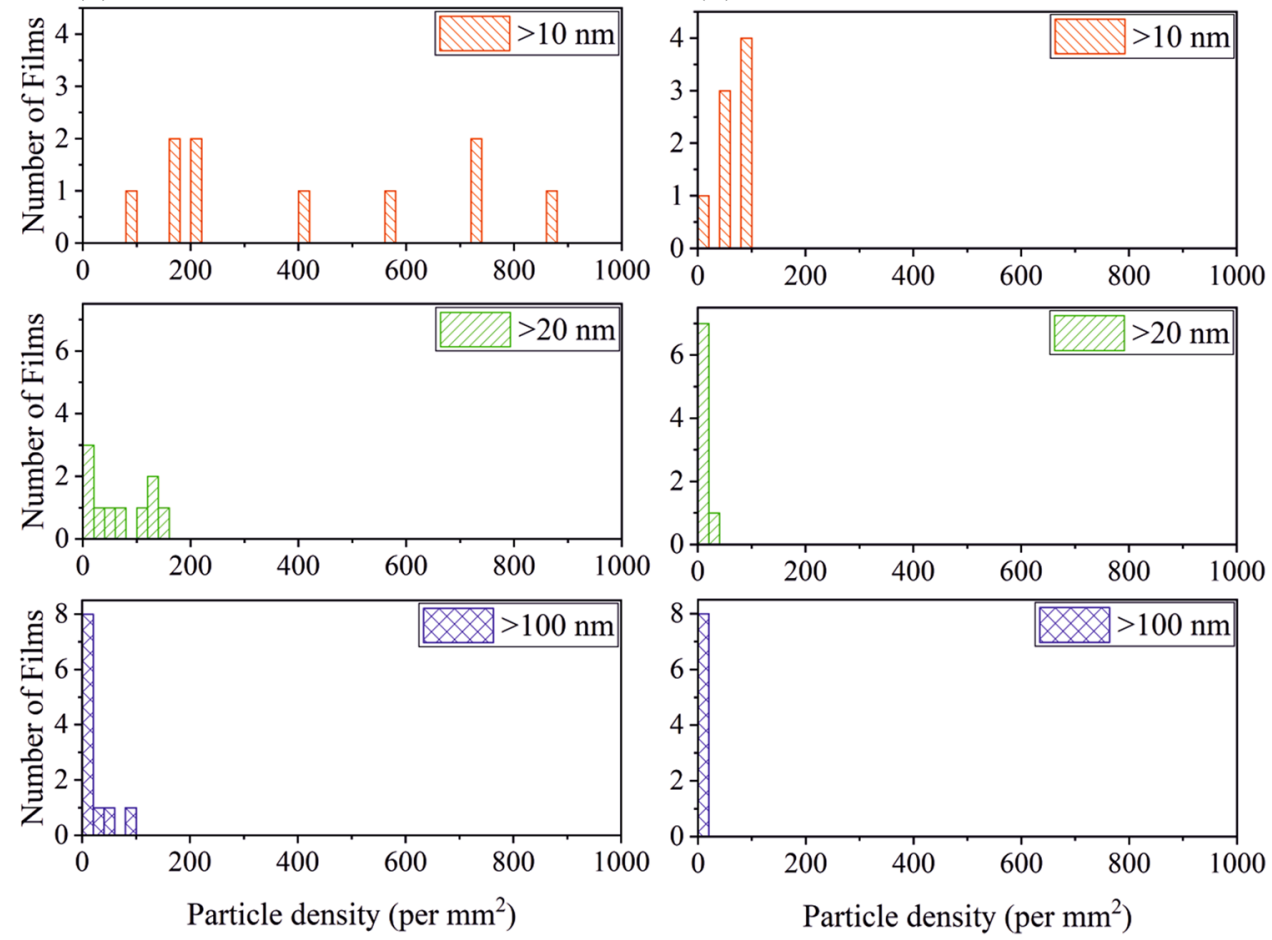

\section{Loss measurements}

A higher density of particulates embedded into planar waveguides has been shown to increase the lasing threshold [11], attributed to an increase in linear propagation loss caused by scattering. To measure the effect of bi-directional ablation on the losses of the resultant films, three Er-doped YGG films were grown to thicknesses of 22, 18 and $13 \mu \mathrm{m}$, at an optimized temperature of $\sim 800{ }^{\circ} \mathrm{C}$ and an optimized ablation fluence of $1.6 \mathrm{~J} / \mathrm{cm}^{2}$, from targets with Er-doping concentrations of 4,4 and 2 at.\%, respectively. The samples were facet-polished and the losses measured via end-coupling of a wavelength-tunable laser, through determination of its spectrally dependent transmission. The internal propagation losses for the three films were then estimated as being 0.17 , 0.36 and $0.17 \mathrm{~dB} / \mathrm{cm}$ for the 22,18 and $13 \mu \mathrm{m}$ thick films respectively. Table 1 shows the range in propagation loss for these 3 films grown with bi-directional ablation, compared to all our previously measured samples grown with the uni-directional approach. The results demonstrate a clear connection between reduced number density of surface particulates and lower propagation loss, which is approaching loss-values achieved via other fabrication methods and is a major improvement with respect to previous PLD-grown waveguides $[5,6]$. This shows great promise for high-efficiency, PLD-grown amplifier devices, suited to high-peakpower and ultra-short laser pulses.
Table 1 Range of loss values and total particulate density (calculated from dark field images) for a range of growths with uni-directional ablation and bi-directional ablation

\begin{tabular}{lll}
\hline Ablation technique & Loss $(\mathrm{dB} / \mathrm{cm})$ & $\begin{array}{l}\text { Total } \\
\text { particulates } \\
\left(/ 100 \mu \mathrm{m}^{2}\right)\end{array}$ \\
\hline Uni-directional & $0.8-7.0$ & $243-1232$ \\
Bi-directional & $0.17-0.36$ & $22-97$ \\
\hline
\end{tabular}

\section{Conclusion}

We have developed a PLD system in which a target is ablated at equal and opposite angles of incidence to the target normal, to reduce the formation of directional surface structure. Using this system of bi-directional ablation, we have shown a $\sim 50 \%$ decrease in surface roughness of the post-ablated target material, in addition to a $\sim 50 \%$ decrease in surface roughness for the corresponding films grown, when compared to uni-directional ablation. Furthermore, the resultant films have $\sim 60 \%$ fewer scattering points embedded into the film, and waveguide propagation losses of $<0.2 \mathrm{~dB} /$ $\mathrm{cm}$ have been realized. The decreased propagation losses of these waveguides grown via our bi-directional ablation protocol will improve the performance of active waveguide devices and lead to the further advancement of novel optical amplifiers. 
Acknowledgements The authors would like to thank Glenn Topley for his mechanical design and fabrication expertise, Ping Hua for her sample processing expertise, and Jim Greer for the permission to include Fig. 7. The authors acknowledge the support of the EPSRC through Grant nos. EP/L021390/1, EP/N018281/1 and EP/J008052/1, the data included in this paper can be found at https://doi.org/10.5258/SOTON /D0722.

Open Access This article is distributed under the terms of the Creative Commons Attribution 4.0 International License (http://creativeco mmons.org/licenses/by/4.0/), which permits unrestricted use, distribution, and reproduction in any medium, provided you give appropriate credit to the original author(s) and the source, provide a link to the Creative Commons license, and indicate if changes were made.

\section{References}

1. R.W. Eason (ed.), Pulsed Laser Deposition of Thin Films (Wiley, Hoboken, 2007)

2. D. Dijkkamp et al., Preparation of Y-Ba-Cu oxide superconductor thin films using pulsed laser evaporation from high Tc bulk material. Appl. Phys. Lett. 51(8), 619-621 (1987)

3. S.H. Waeselmann et al., Lasing of $\mathrm{Nd}^{3+}$ in sapphire. Laser Photonics Rev. 10(3), 510-516 (2016)

4. A.A. Anderson et al., Ti:sapphire planar waveguide laser grown by pulsed laser deposition. Opt. Lett. 22(20), 1556-1557 (1997)

5. S.J. Beecher et al., Diode-end-pumped $1.2 \mathrm{~W}$ Yb: $\mathrm{Y}_{2} \mathrm{O}_{3}$ planar waveguide laser. Opt. Express 22(18), 22056-22061 (2014)

6. T.L. Parsonage et al., Pulsed laser deposited diode-pumped $7.4 \mathrm{~W}$ $\mathrm{Yb}: \mathrm{Lu}_{2} \mathrm{O}_{3}$ planar waveguide laser. Opt. Express 23(25), 3169131697 (2015)

7. J.A. Grant-Jacob et al., Dynamic control of refractive index during pulsed-laser-deposited waveguide growth. Opt. Mater. Express 7(11), 4073 (2017)

8. J. Mackenzie et al., Functional crystal films fabricated by pulsed laser deposition, in PHOTONICS International Congress 2018. Yokohama, Japan, (2018)
9. S.V. Kurilchik et al., Pulsed-laser-deposited Yb:YAG planarwaveguide amplifier, in 8th EPS-QEOD Europhoton. Barcelona, Spain, (2018)

10. S.J. Beecher et al., Ytterbium-doped-garnet crystal waveguide lasers grown by pulsed laser deposition. Opt. Mater. Express 7(5), 1628 (2017)

11. S.J. Barrington et al., The effect of particulate density on performance of $\mathrm{Nd}: \mathrm{Gd}_{3} \mathrm{Ga}_{5} \mathrm{O}_{12}$ waveguide lasers grown by pulsed laser deposition. Opt. Commun. 185(1-3), 145-152 (2000)

12. J.A. Grant-Jacob et al., Particulate reduction in ternary-compound film growth via pulsed laser deposition from segmented binarytargets. Mater. Res. Express 5(3), 036402 (2018)

13. P.K. Schenck et al., Particulate reduction in the pulsed laser deposition of barium. Appl. Surf. Sci. 127(129), 655-661 (1998)

14. T.P. O'Brien et al., The effect of laser fluence on the ablation and deposition of $\mathrm{YBa}_{2} \mathrm{Cu}_{3} \mathrm{O}_{7}$. Mater. Sci. Eng. B 13(1), 9-13 (1992)

15. A. Marcu et al., Pulsed laser deposition of YBCO thin films in a shadow mask configuration. Thin Solid Films 360, 166-172 (2000)

16. E.V. Pechen et al., Pulsed-laser deposition of smooth high-Tc superconducting films using a synchronous velocity filter. Appl. Phys. Lett. 66(17), 2292-2294 (1995)

17. T. Yoshitake et al., Elimination of droplets using a vane velocity filter for pulsed laser ablation of $\mathrm{FeSi}_{2}$. Appl. Surf. Sci. 197, 379-383 (2002)

18. A. Gorbunoff et al., The cross-beam pulsed laser deposition, in Photon Processing in Microelectronics and Photonics V 2006. International Society for Optics and Photonics (2006)

19. J.A. Greer et al., Large-area pulsed laser deposition-techniques and applications. J. Vac. Sci. Technol. A. 13(3), 1175-1181 (1995)

Publisher's Note Publisher's Note Springer Nature remains neutral with regard to jurisdictional claims in published maps and institutional affiliations. 